\title{
HOPFIAN BOOLEAN ALGEBRAS OF POWER LESS THAN OR EQUAL TO CONTINUUM
}

JAMES T. LOATS

\begin{abstract}
An infinite Boolean algebra (BA) is said to be hopfian if every onto endomorphism is one-to-one. It is shown that there are no countably infinite hopfian BA's. An atomic hopfian BA of power continuum is constructed.
\end{abstract}

We restrict our attention to infinite Boolean algebras $\mathfrak{A}=$ $(A,+, \cdot,-, 0,1)$, and use End $\mathfrak{A}$ for the endomorphism semigroup of $\mathfrak{A}$, $\operatorname{At}(\mathfrak{U})$ for the set of atoms of $\mathfrak{A}$, and $\operatorname{Sg}(T)$ for the subalgebra of $\mathfrak{A}$ generated by $T \subseteq A \cdot \mathscr{P}(\omega)$ denotes the power set of $\omega$ and $\mathbb{E}(\omega)$, the field of finitecofinite subsets of $\omega$. We use $\operatorname{MA}(\kappa)$ to denote Martin's axiom: If $\mathcal{P}$ is a partial ordering satisfying the countable antichain condition, and if $\mathscr{F}$ is a collection of dense open subsets of $\mathscr{P}$ with $|\mathscr{F}| \leqslant \kappa$, then there is an $\mathscr{F}$-generic filter on $\mathcal{P}$. (See $A_{\kappa}$ of Martin and Solovay [2].) Recall that $\operatorname{MA}\left(\aleph_{0}\right)$ is a theorem in $\mathrm{ZFC}$.

THEOREM 1. Let $\mathfrak{A}$ be a $B A$ of power $\kappa<2^{\kappa_{0}}$ with infinitely many atoms and assume $\mathrm{MA}(\kappa)$ holds. Then there is $\mathfrak{B} \subseteq \mathfrak{A}$ such that $\mathfrak{B} \cong \mathfrak{E}(\omega)$ and there is an isomorphism ${ }^{+}$: End $\mathfrak{B} \rightarrow$ End $\mathfrak{A}$ such that, for $h \in$ End $\mathfrak{B}, h$ is one-to-one (onto) iff $h^{+}$is one-to-one (onto, respectively).

The following lemma, which is (1) of Theorem 3.6 of McKenzie and Monk [3], is proved using $\operatorname{MA}(\kappa)$.

Lemma 2. Under the hypothesis of Theorem 1, there is a $D \subseteq \operatorname{At}(\mathfrak{A})$, with $|D|=\aleph_{0}$ such that for every $a \in A$, the set $S(a)=\{d \in D \mid d \leqslant a\}$ is a finite or cofinite subset of $D$.

Proof of Theorem 1. Let $\mathfrak{B}=\operatorname{Sg}(D)$ and notice $\mathfrak{B} \cong \mathfrak{S}(\omega)$. Clearly, $I=\{a \in A \mid S(a)$ is finite $\}$ is a maximal ideal of $\mathfrak{A}$. For $a \in I$, there is a unique finite $S(a) \subseteq D$ and unique $t(a) \in A$ such that $a=\Sigma S(a)+t(a)$ and $d \cdot t(a)=0$ for all $d \in D$.

Let $h \in$ End $\mathfrak{B}$. For each $a \in I$, define

$$
f(a)=\sum\{h(d) \mid d \in S(a)\}+t(a) .
$$

Presented to the Society, August 17, 1977 under the title On 'strongly' rigid Boolean algebras and cofinalities of Boolean algebras; received by the editors October 24, 1978 and, in revised form, November 29, 1978.

AMS (MOS) subject classifications (1970). Primary 06A40, $02 \mathrm{~J} 05$.

Key words and phrases. Hopfian Boolean algebra.

(C) 1979 American Mathematical Society 0002-9939/79/0000-0505/\$02.25 
Define $h^{+}: \mathfrak{A} \rightarrow \mathfrak{U}$ by

$$
h^{+}(a)= \begin{cases}f(a) & \text { if } a \in I, \\ -f(-a) & \text { if } a \notin I .\end{cases}
$$

It is easy to check that $h^{+}$is an endomorphism of $\mathfrak{A}$ satisfying Theorem 1 .

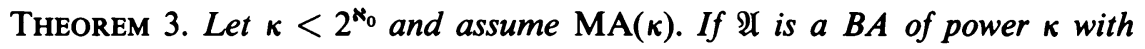
infinitely many atoms, then $\mathfrak{A}$ is not hopfian.

Proof. By Theorem 1, it suffices to note that $\Subset(\omega)$ is not hopfian. In fact, it is well known that any finite-to-one map $t: \omega \rightarrow \omega$ (i.e., $t^{-1}(\{i\})$ is finite for all $i \in \omega)$ induces an endomorphism $\hat{t}$ of $\mathbb{(}(\omega)$ via $\hat{t}(s)=\{i \mid t(i) \in s\}$. It is easy to check that $t$ is one-to-one (onto) iff $\hat{t}$ is onto (one-to-one). An appropriate choice of $t$ finishes the proof.

COROLlaRy 4. There are no countable hopfian Boolean algebras.

Proof. Let $\mathfrak{A}$ be countably infinite. By Theorem 3, we may assume that $\mathfrak{A}$ has only finitely many atoms with sum $s$. So $\mathfrak{A} \cong \mathfrak{A} \uparrow s \times \mathfrak{U} \uparrow-s$ where $\mathfrak{A} \uparrow-s$ is isomorphic to the countable atomless BA $\mathfrak{F}$. Let $\left\{i_{n}: n \in \omega\right\}$ be the independent generators of $\mathfrak{F}$. Then $h_{1}$ defined by $h_{1}\left(i_{0}\right)=h\left(i_{1}\right)=i_{0}$ and $h_{1}\left(i_{n+1}\right)=i_{n}$ for $n>0$ can be extended to an endomorphism $h$ of $\mathfrak{F}$ which is onto but not one-to-one. When "attached" to the identity on $\mathfrak{A} \uparrow s, h$ clearly demonstrates that $\mathfrak{A}$ is not hopfian.

There is a nice translation of some of the above into topological terms, as was pointed out by the referee. In this paragraph, consider only BA's with infinitely many atoms. For such an algebra $\mathfrak{A}$, let $X$ be its Stone space. The conclusion of Lemma 2 is easily seen to be equivalent to the following property denoted by (*): There is a countably infinite subset $D$ of $X$ of isolated points such that the closure $\bar{D}$ of $D$ in $X$ has $|\bar{D} \sim D|=1$. Every superatomic BA (every subalgebra is atomic) and every BA with an ordered base satisfies (*). In particular, every countable BA satisfies (*). Obviously, if $\mathfrak{A}$ satisfies (*), then $\mathfrak{A}$ is not hopfian. For if $D=\left\{d_{n}: n \in \omega\right\}$, define $f$ : $X \rightarrow X$ by $f\left(d_{n}\right)=d_{n+1}$ and $f(x)=x$ for $x \in X \sim D$. Then $f$ is continuous, one-to-one, and not onto.

The results above also hold for BA's which might be called dual hopfian; i.e. every one-to-one endomorphism is onto. The proofs require little modification.

In Theorem 3, the requirement that $\mathfrak{A}$ have infinitely many atoms is necessary when $\mathfrak{A}$ is uncountable, since there are atomless (in fact, rigid) hopfian BA's in every uncountable cardinality. (See Loats and Rubin [1].) Also, note that Corollary 4 and the results in [1] yield a complete description of the cardinalities in which atomless hopfian BA's can occur.

We now turn to the construction of an atomic hopfian BA of power $2^{\kappa_{0}}$. 
LeMMA 5. Let $\left\{d_{n} \mid n \in \omega\right\}$ be a family of pairwise disjoint nonempty subsets of $\omega$ satisfying

( $\nabla):$ If $\left|d_{n}\right|=1$, for all $n \in \omega$, then there is an infinite set $S \subseteq \omega$ such that $n \in S$ iff $d_{n} \neq\{n\}$. Then there is an infinite $M \subseteq \omega$ such that

$$
M \cap \bigcup_{n \in M} d_{n}=\varnothing .
$$

Proof. Case 1. $\left|d_{n}\right|=1$ for all $n \in \omega$. Obvious.

Case 2. There is $n_{0}$ with $\left|d_{n_{0}}\right|>1$. By induction, choose $n_{1} \in d_{n_{0}} \sim\left\{n_{0}\right\}$, and for $k>1$, choose $n_{k+1} \in d_{n_{k}}$ such that $n_{k+1} \neq n_{1}$ if $n_{k}=n_{0}$. By straightforward induction on $j$ one shows that $1 \leqslant i<j$ implies $n_{i} \neq n_{j}$. Let $M=$ $\left\{n_{2 i} \mid 0 \neq i \in \omega\right\}$.

THEOREM 6. There is an atomic hopfian $B A \mathfrak{A} \subseteq \mathscr{P}(\omega)$ of power $2^{\mathrm{K}_{0}}$ such that $\mid$ Aut $\mathfrak{U} \mid=\boldsymbol{\aleph}_{0}$.

Proof. For each $\alpha<2^{\aleph_{0}}$, let $\left\langle d_{n}^{\alpha}: n \in \omega\right\rangle$ be a sequence of pairwise disjoint nonempty subsets of $\omega$ satisfying $(\nabla)$ of Lemma 5. Furthermore, assume that every infinite partition of $\omega$ and each of its permutations appear $2^{\aleph_{0}}$ times in the sequence $\left\langle\left\langle d_{n}^{\alpha}: n \in \omega\right\rangle: \alpha\left\langle 2^{\aleph_{0}}\right\rangle\right.$.

Let $\mathfrak{A}_{0}=\Subset(\omega)$ and set $B_{0}=\varnothing$. We shall define $\mathfrak{A}_{\alpha}, B_{\alpha}$ for each $\alpha<2^{\aleph_{0}}$ so that

$$
\left|A_{\alpha}\right|,\left|B_{\alpha}\right|<|\alpha|+\aleph_{0}
$$

and

$$
A_{\alpha} \cap B_{\alpha}=\varnothing .
$$

For a limit ordinal $\lambda<2^{\kappa_{0}}$, set

$$
\mathfrak{A}_{\lambda}=\bigcup_{\alpha<\lambda} \mathfrak{A}_{\alpha} \text { and } B_{\lambda}=\bigcup_{\alpha<\lambda} B_{\alpha} .
$$

Let $\alpha<2^{\aleph_{0}}$. In order to define $\mathfrak{A}_{\alpha+1}$ and $B_{\alpha+1}$, consider $\left\langle d_{n}^{\alpha}: n \in \omega\right\rangle$. For convenience, we suppress the index $\alpha$. If $\left\langle d_{n}: n \in \omega\right\rangle \notin A_{\alpha}$, then set $\mathfrak{A}_{\alpha+1}=$ $\mathfrak{A}_{\alpha}$ and $B_{\alpha+1}=B_{\alpha}$. So assume that $\left\{d_{n}: n \in \omega\right\} \subseteq A_{\alpha}$. By Lemma 5 , there is an infinite $M \subseteq \omega$ such that $M \cap \cup_{n \in M} d_{n}=\varnothing$. Set

$$
D_{\alpha}=\left\{\bigcup_{n \in S} d_{n} \mid S \subseteq M\right\} .
$$

For each $T \in D_{\alpha}$, set $f_{\alpha}(T)=\left\{n \mid d_{n} \subseteq T\right\}$. Notice $T \cap f_{\alpha}(T)=\varnothing$ for all $T \in D_{\alpha}$. We shall show:

(1) There is $J \in D_{\alpha} \sim A_{\alpha}$ such that

$$
\operatorname{Sg}\left(A_{\alpha} \cup\{J\}\right) \cap\left(B_{\alpha} \cup\left\{f_{\alpha}(J)\right\}\right)=\varnothing .
$$

For if (1) holds, set

$$
\begin{aligned}
\mathfrak{A}_{\alpha+1} & =\operatorname{Sg}\left(A_{\alpha} \cup\{J\}\right), \\
B_{\alpha+1} & =B_{\alpha} \cup\left\{f_{\alpha}(J)\right\}
\end{aligned}
$$


and proceed by induction. But if not, we have:

(2) For every $J, J \in D_{\alpha} \sim A_{\alpha}$, there exist $C, D \in A_{\alpha}$ such that

$$
(C \cap J) \cup(D \sim J) \in B_{\alpha} \cup\left\{f_{\alpha}(J)\right\} \text {. }
$$

Let $K_{0} \subseteq D_{\alpha}$ be of power $2^{\aleph_{0}}$ such that for $J_{0}, J_{1} \in K_{0}, f_{\alpha}\left(J_{0}\right) \cap f_{\alpha}\left(J_{1}\right)$ is a finite subset of $\omega$. By (2), we have

$$
K_{0} \sim A_{\alpha}=\bigcup_{C, D \in A_{\alpha}}\left\{J \in K_{0} \sim A_{\alpha} \mid(C \cap J) \cup(D \sim J) \in B_{\alpha} \cup\left\{f_{\alpha}(J)\right\}\right\} .
$$

Since $\left|A_{\alpha}\right|,\left|B_{\alpha}\right|<2^{\aleph_{0}}$ and $\left|K_{0} \sim A_{\alpha}\right|=2^{\aleph_{0}}$, there exist $C, D \in A_{\alpha}$ such that for

$$
K_{1}=\left\{J \in K_{0} \sim A_{\alpha} \mid(C \cap J) \cup(D \sim J) \in B_{\alpha} \cup\left\{f_{\alpha}(J)\right\}\right\}
$$

we have $\left|K_{1}\right|>\left|A_{\alpha}\right|+\left|B_{\alpha}\right|$. So

(3) There is at most one $J \in K_{1}$ such that $(C \cap J) \cup(D \sim J)=f_{\alpha}(J)$.

For if not, let $J_{0}, J_{1}$ be two such $J$ 's. Since $J_{i} \cap M=\varnothing$ and $f_{\alpha}(J) \subseteq M$, it is easy to see that $f_{\alpha}\left(J_{i}\right)=D \sim J_{i}$. In fact, one can even show for $i=0,1$, $f_{\alpha}\left(J_{i}\right)=D \cap M$. But since $J_{0}$ and $J_{1}$ are distinct, so are $f_{\alpha}\left(J_{0}\right)$ and $f_{\alpha}\left(J_{1}\right)$, a contradiction.

Let

$$
K_{2}=\bigcup_{E \in B_{\alpha}}\left\{J \in K_{1} \mid(C \cap J) \cup(D \sim J)=E\right\}
$$

By (3), $\left|K_{2}\right|>\left|B_{\alpha}\right|$, so there is $E \in B_{\alpha}$ such that for

$$
K_{3}=\left\{J \in K_{2} \mid(C \cap J) \cup(D \sim J)=E\right\},
$$

we have $\left|K_{3}\right|>\left|B_{\alpha}\right|+\aleph_{0}$. In particular, we may choose distinct $J, H \in K_{3}$. Now

$$
E=[C \cap J \cap H] \cup[C \cap D] \cup[D \sim(H \cap J)] .
$$

Since $J \cap H \in A_{\alpha}$ as are $C, D$, we have $E \in A_{\alpha} \cap B_{\alpha}$, a contradiction. Therefore, (1) holds. Next,

(4) $\mathfrak{A}=\mathfrak{A}_{2^{\mathfrak{n}_{0}}}$ is the desired BA. For, let $h \in$ End $\mathfrak{A}$ such that

(a) $h$ is onto, and

(b) $h$ is not an automorphism of $\mathfrak{A}$ induced by a permutation of At $\mathfrak{A}$ with finite support.

By induction, we choose a family $\left\langle d_{n}: n \in \omega\right\rangle$ of pairwise disjoint nonempty subsets of $\omega$ so that $h\left(d_{n}\right)=\{n\}$ for all $n$. In case $h$ is not one-to-one, it is clear that we may insure that at least one $d_{n}$ has two or more elements. It remains to check that $\left\langle d_{n}: n \in \omega\right\rangle$ satisfies $(\nabla)$ : By our choice above, if $\left|d_{n}\right|=1$ for all $n$, then $h$ is an automorphism. By condition (b), there must exist an infinite $S \subseteq \omega$ such that $h \in S$ iff $d_{n} \neq\{n\}$, as desired.

Since $c f\left(2^{\aleph_{0}}\right)>\aleph_{0}$, there is $\alpha<2^{\aleph_{0}}$ such that

$$
\left\langle d_{n}: n \in \omega\right\rangle=\left\langle d_{n}^{\alpha}: n \in \omega\right\rangle \subseteq A_{\alpha} .
$$

At stage $\alpha$ (see (1)), we put a $J \in D_{\alpha}$ into $A_{\alpha+1}$ so that $f_{\alpha}(J) \notin A$. But 
$f_{\alpha}(J)=h(J)$. For if $d_{n} \subseteq J$, then $\{n\}=h\left(d_{n}\right) \subseteq h(J)$. If $d_{n} \& J$, then $d_{n} \cap J$ $=\varnothing$. So $\{n\} \cap h(J)=\varnothing$. Thus $f_{\alpha}(J)=h(J) \notin A$, a final contradiction.

Problem 7. Are there any atomic hopfian BA's with uncountably many atoms?

ACKNOWLEDGEMENT. The preceding constitutes a portion of the author's Ph.D. thesis at the University of Colorado. The author expresses his deep appreciation to his advisor, Professor J. Donald Monk for his patience and encouragement.

ADDED IN PROOF. I have recently noticed that the proof of Theorem 6 may be modified to obtain an atomic Boolean algebra of power $2^{\kappa_{0}}$ which is dual hopfian. In fact, the two constructions can be merged to obtain an algebra which is both hopfian and dual hopfian.

Judy Roitman and myself have recently shown that it is consistent with $\mathrm{ZFC}+\operatorname{not} \mathrm{CH}$ that the answer to Problem 7 is yes.

\section{REFERENCES}

1. J. Loats and M. Rubin, Boolean algebras without nontrivial onto endomorphisms exist in every uncountable cardinality, Proc. Amer. Math. Soc. 72 (1978), 346-351.

2. D. Martin and M. Solovay, Internal Cohen extensions, Ann. Math. Logic 2 (1970), 143-178. MR 42 \# 5787.

3. R. McKenzie and J. D. Monk, On automorphism groups of Boolean algebras, Colloq. Math. Publ. 10 (1973), 951-988. MR 51 \# 12651.

Department of Mathematics, University of Kansas, LAWREnCe, Kansas 66045 